\title{
CONTACT INTERACTION OF RIGID STAMP AND INFINITE ORTHOTROPIC PLATE WITH CLOSE TO ELLIPTICAL HOLE
}

\author{
Andriy KOTSYUBA \\ *Lutsk National Technical University, Lvivska Str. 75, 43018 Lutsk, Ukraine \\ akaerkaj@rambler.ru
}

\begin{abstract}
Numerical method of finding the contact stresses under the stamp of complex shape for the case of orthotropic plates with close to an elliptical hole, which based on constructed in Bozhydarnik et al., (2007) algorithm, is developed. The distribution of contact stresses under the stamp, which shape matches the shape of the hole, is investigated.
\end{abstract}

Key words: Orthotropic, Elliptical Hole, Numerical Methot, Contact Stresses

\section{INTRODUCTION}

The problem of contact interaction of rigid smooth stamp of complex shape with elliptic boundary close to the hole (with the semi-axis $R_{x}$ and $R_{y}$ ) in orthotropic infinite plate is examined. This problem sufficiently for such cases is studied: stamp has a circular shape and is close in size to the hole (Abd-El-Naby et al., 1993; Kombel, 2003; Panasyuk and Tepliy, 1975; Savin, 1968); stamp or system of stamps with corner points (Syackyj and Shynkarchuk, 2010; Syackyj and Trohymchuk, 2009, 2010a); infinite plate has elliptical (Bozhydarnik et al., 2007; Kombel, 2003; Sawin, 1968; Syackyj and Shynkarchuk, 2010, Syackyj and Trohymchuk, 2009) or more complex forms hole (Maksimovic et al., 2012; Syackyj and Shynkarchuk, 2011; Syackyj and Trohymchuk, 2010a, b); the contour of the curved hole infinite plate is partially strengthened (Syackyj and Trohymchuk, 2009, 2010b) etc.

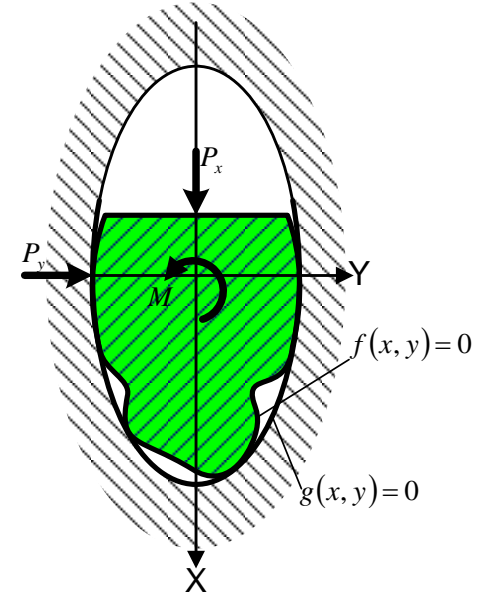

Fig. 1. Problem

Developed in this paper, a numerical method allows solving flat contact problems of elasticity theory in which the contact area is unknown in advance and may include individual sites.

We assume that the plate is weakened by close to an elliptical hole. Let the equation of the path that limits the hole, is given in implicit form $g(x, y)=0$ (or parametric $-x=g_{1}(\alpha), y=$ $g_{2}(\alpha)$ and the border punch to the contact interaction - the equation:

$f(x, y)=0$

Suppose that known forces applied to the piston head and moment vectors are equal $\left(P_{x}, P_{y}\right)$ and $M$.

While solving, we have that between the stamp and orthotropic body there are frictional forces. Assume that the stamp is in a state of limited equilibrium and there is the Coulomb's-Amontons law.

To solve the problem we use the numerical-analytic method (Bozhydarnik et al., 2007), which is based on the method of integral equations of mechanical quadratures and quadratic programming.

\section{CONTACT CONDITIONS}

Assuming that after the contact interaction shifting of the stamp in the direction of the coordinate axis $O x$ in the distance $D_{x}$ and the angle of its rotation $\theta$, we obtain from Bozhydarnik et al., (2007) terms of the contact form:

$$
\begin{aligned}
& \begin{array}{l}
f_{1}(\alpha) \tilde{u}(\alpha)+f_{2}(\alpha) \tilde{v}(\alpha)=-\tilde{f}(\alpha)-D_{x} f_{1}(\alpha) \\
+\theta f_{3}(\alpha)+\delta(\alpha)
\end{array} \\
& \text { where: } \tilde{f}(\alpha)=f\left(g_{1}(\alpha), g_{2}(\alpha)\right) ; \\
& f_{1}(\alpha)=f_{x}^{\prime}\left(g_{1}(\alpha), g_{2}(\alpha)\right) ; \\
& f_{2}(\alpha)=f_{y}^{\prime}\left(g_{1}(\alpha), g_{2}(\alpha)\right) ; \\
& f_{3}(\alpha)=g_{1}(\alpha) \cdot f_{2}(\alpha)-g_{2}(\alpha) \times \quad \times f_{1}(\alpha) ; \\
& \tilde{u}(\alpha)=u\left(g_{1}(\alpha), g_{2}(\alpha)\right) ; \\
& \tilde{v}(\alpha)=v\left(g_{1}(\alpha), g_{2}(\alpha)\right)
\end{aligned}
$$

plate boundaries due to the influence of an applied thereto a stamp; displacements $-\delta(\alpha)=o(\tilde{u}, \tilde{v})$ - additional members that have another order of smallness relative displacements. 
Let the tangent $\tau$ at the stamp associated with the normal $\sigma$ according to the Coulomb's-Amontons law $-\sigma=\rho \tau \quad(\rho$ - coefficient of friction), whereas in the case of an elliptical hole axis $R_{x}$ and $R_{y}$ and the value for the displacement can be presented as:

$\left\{\begin{array}{l}\tilde{u}(\alpha)=\int_{\Omega} \sigma(\xi)\left(K_{1}(\alpha, \xi)-\rho K_{2}(\alpha, \xi)\right) d \xi \\ \tilde{v}(\alpha)=\int_{\Omega} \sigma(\xi)\left(K_{2}(\alpha, \xi)-\rho K_{1}(\alpha, \xi)\right) d \xi\end{array}\right.$

where $\Omega$ - a set of segments along which there is contact;

$$
\left\{\begin{aligned}
K_{1}(\alpha, \xi)= & -\frac{1}{\pi}\left[-n_{1} \ln 2 \sin \frac{|\alpha-\xi|}{2} R_{y} \cos \xi\right. \\
& \left.-n_{2} \frac{\alpha-\xi+\pi}{2} R_{x} \sin \xi\right] \\
K_{2}(\alpha, \xi)= & -\frac{1}{\pi}\left[-n_{3} \ln 2 \sin \frac{|\alpha-\xi|}{2} R_{x} \sin \xi\right. \\
& \left.+n_{2} \frac{\alpha-\xi+\pi}{2} R_{y} \cos \xi\right]
\end{aligned}\right.
$$

where: $\quad n_{1}=\frac{\beta_{1}+\beta_{2}}{E_{1}}, \quad n_{2}=\frac{-v_{12}}{E_{1}}+\frac{1}{\beta_{1} \beta_{2} E_{2}}=\frac{-v_{12}}{E_{1}}+\frac{\beta_{1} \beta_{2}}{E_{1}}=$ $\frac{-v_{21}}{E_{2}}+\frac{1}{\beta_{1} \beta_{2} E_{2}}=\frac{-v_{21}}{E_{2}}+\frac{\beta_{1} \beta_{2}}{E_{1}}, n_{3}=\frac{\beta_{1}+\beta_{2}}{E_{2} \beta_{1} \beta_{2}}$; here $E_{1}, E_{2}$ i $v_{12}$, $v_{21}$ - the Young's modulus and the Poisson's ratio for the axes Ox and $O y ; \beta_{1}$ i $\beta_{2}$ - positive roots of the characteristic biquadratic equation:

$\frac{\beta^{4}}{E_{1}}+\left(-\frac{2 v_{12}}{E_{1}}+\frac{1}{G}\right) \beta^{2}+\frac{1}{E_{2}}=0$

where $G$ - the shear modulus.

Further, we suppose that the hole is close to the semi-axis $R_{x}$ and $R_{y}$ of the elliptical shape. Then its equation in parametric form is: $g_{1}(\alpha)=\tilde{R}_{x}(\alpha) \cos \alpha, g_{2}(\alpha)=\tilde{R}_{y}(\alpha) \sin \alpha, \alpha \in[-\pi, \pi)$, where $R_{x}-\tilde{R}_{x}(\alpha)=g_{x \varepsilon}(\alpha), R_{y}-\tilde{R}_{y}(\alpha)=g_{y \varepsilon}(\alpha)$. Here $g_{x \varepsilon}$ and $g_{y \varepsilon}$ - limited by the absolute small number $\varepsilon$ of features that characterize the deviation from the elliptical shape hole. We consider that the size $\varepsilon$ is quite small, and in its relationships for displacements can be neglected without significant effect on the distribution of the desired contact stresses, i.e., moving on the surface of the hole set relations (3).

Additional equality for finding unknown $D_{x}$ and $\theta$ looks like:

$\left\{\begin{array}{l}\int_{\Omega}(\xi)\left(\tilde{R}_{y}(\xi) \cos \xi-\rho \tilde{R}_{x}(\xi) \sin \xi\right) d \xi=P_{x} \\ \int_{\Omega}(\xi)\left(\tilde{R}_{x}(\xi) \sin \xi-\rho \tilde{R}_{y}(\xi) \cos \xi\right) d \xi=P_{y}\end{array}\right.$

\section{CONSTRUCTION OF INTEGRAL EQUATIONS}

Let the stamp has a form close to elliptical with the semi-axis of the $r_{x}$ and $r_{y}$. Then its equation is possible to present as the parametric form: $x=\tilde{r}_{x}(\alpha) \cos \alpha, \quad y=\tilde{r}_{y}(\alpha) \sin \alpha, \quad \alpha \in$ $[-\pi, \pi)$, where $r_{x}-\tilde{r}_{x}(\alpha)=f_{x \delta}(\alpha), r_{y}-\tilde{r}_{y}(\alpha)=f_{y \delta}(\alpha)$. Here $f_{x \delta}$ and $f_{y \delta}$ - limited by the absolute small number of features $\delta$ that characterize the deviation from the elliptical shape stamp.

To construct the integral equation (2) it was necessary to find the appropriate function $f$, i.e. an equation for hard punch in implicit form (1). If we draw considerations for arbitrary functions $\tilde{r}_{x}$ and $\tilde{r}_{y}$, then it is very much complicate further calculations. Therefore, to simplify, we assume that $f_{x \delta}(\alpha)=r_{x} \delta \cos (k \alpha)$ and $f_{y \delta}(\alpha)=r_{y} \delta \cos (k \alpha)$. Then the equation stamp (1) has the form:

$$
(x-\varepsilon)^{2}+m y^{2}-r_{x}^{2}(1-\delta \cos (k \hat{\alpha}(x, y)))^{2}=0,
$$

$$
\text { where } m=\frac{r_{x}^{2}}{r_{y}^{2}} ; \hat{\alpha}(x, y)=\left\{\begin{array}{l}
\arctan \left(\frac{r_{x} y}{r_{y} x}\right), \quad x>0 ; \\
\arctan \left(\frac{r_{x} y}{r_{y} x}\right)+\pi, x<0, y \geq 0 ; \\
\arctan \left(\frac{r_{x} y}{r_{y} x}\right)-\pi, x<0, y<0 ; \\
\frac{\pi}{2} \operatorname{sgn} y, \quad x=0, y \neq 0 .
\end{array}\right.
$$

Ignoring the size $\delta$, hold linearization of integrated equation (2) for a hard punch whose shape is given by equation (5). Then in condition (2) function $\tilde{f}, \tilde{f}_{1}, \tilde{f}_{2}$ i $\tilde{f}_{3}$, we obtain as a:

$$
\begin{aligned}
& \tilde{f}(\alpha)=\tilde{R}_{x}^{2}(\alpha)-r_{x}^{2}(1-\delta \cos (k \tilde{\alpha}(\alpha)))^{2}+\varepsilon^{2}-2 \tilde{R}_{x}(\alpha) \varepsilon \cos \alpha+ \\
& +\left(m \tilde{R}_{y}^{2}(\alpha)-\tilde{R}_{x}^{2}(\alpha)\right) \sin ^{2} \alpha, \\
& \tilde{f}_{1}(\alpha)=2\left(\tilde{R}_{x}(\alpha) \cos \alpha-\varepsilon\right)- \\
& -r_{x}^{2} \delta k(1-\delta \cos (k \tilde{\alpha}(\alpha))) \sin (k \tilde{\alpha}(\alpha)) \tilde{\alpha}_{1}(\alpha), \tilde{f}_{2}(\alpha)=2 m \times \\
& \times \tilde{R}_{y}(\alpha) \sin \alpha-r_{x}^{2} \delta k(1-\delta \cos (k \tilde{\alpha}(\alpha))) \sin (k \tilde{\alpha}(\alpha)) \tilde{\alpha}_{2}(\alpha), \\
& \tilde{f}_{3}(\alpha)=2 \tilde{R}_{x}(\alpha) \tilde{R}_{y}(\alpha) \sin \alpha \cos \alpha(m-1)+2 \tilde{R}_{y}(\alpha) \varepsilon \sin \alpha+ \\
& +r_{x}^{2} \delta k(1-\delta \cos (k \tilde{\alpha}(\alpha)))\left(\tilde{R}_{y}(\alpha) \tilde{\alpha}_{1}(\alpha) \sin \alpha-\tilde{R}_{x}(\alpha) \tilde{\alpha}_{2}(\alpha) \times\right. \\
& \times \cos \alpha) \sin (k \tilde{\alpha}(\alpha)) ;
\end{aligned}
$$

where

$$
\begin{aligned}
& \tilde{\alpha}(\alpha)=\hat{\alpha}\left(\tilde{R}_{x}(\alpha) \cos \alpha, \tilde{R}_{y}(\alpha) \sin \alpha\right), \\
& \tilde{\alpha}_{1}(\alpha)=\hat{\alpha}_{x}^{\prime}\left(\tilde{R}_{x}(\alpha) \cos \alpha, \tilde{R}_{y}(\alpha) \sin \alpha\right), \\
& \tilde{\alpha}_{2}(\alpha)=\hat{\alpha}_{y}^{\prime}\left(\tilde{R}_{x}(\alpha) \cos \alpha, \tilde{R}_{y}(\alpha) \sin \alpha\right) .
\end{aligned}
$$

After substituting relations for displacements (3) and functions (6) in the linearized form of condition (2), we obtain the integral equation in the form:

$\int_{\Omega} \sigma(\xi) J(\alpha, \xi) d \xi+D_{x} f_{1}(\alpha)-\theta f_{3}(\alpha)=-\tilde{f}(\alpha)$,

where $J(\alpha, \xi)$ is the core of the integral equation and it looks quite bulky.

\section{THE NUMERICAL ALGORITHM FOR SOLVING THE PROBLEM (Syackyj and Shynkarchuk, 2011; Maksimovic et al., 2012)}

Determination of contact stresses of these equations is quite a challenge, because there may be a contact on a few areas, finding of the boundaries is reduced to finding the solutions of nonlinear equations. Solving this problem in the article carried out numerically. We introduce into consideration the area $D=[a, b]$, that contains all of the contact area, and additionally define in it out of the contact areas (at $\alpha \notin \Omega) \sigma(\alpha)=0$. Then 
conditions $(7,4)$ can be written as a system of equationsinequalities:

$$
\left\{\begin{array}{l}
\int_{D} \sigma(\alpha) J(\alpha, \xi) d \xi+D_{x} f_{1}(\alpha)-\theta f_{3}(\alpha)=F(\alpha), \sigma(\alpha)<0, \\
\int_{D} \sigma(\alpha) J(\alpha, \xi) d \xi+D_{x} f_{1}(\alpha)-\theta f_{3}(\alpha) \leq F(\alpha), \sigma(\alpha)=0 \\
\int_{D} \sigma(\xi)\left(\tilde{R}_{y}(\xi) \cos \xi-\rho \tilde{R}_{x}(\xi) \sin \xi\right) d \xi=P_{x} \\
\int_{D} \sigma(\xi)\left(\tilde{R}_{x}(\xi) \sin \xi+\rho \tilde{R}_{y}(\xi) \cos \xi\right) d \xi=P_{y}
\end{array}\right.
$$

where $F(\alpha)=-\tilde{f}(\alpha)$.

The function $J(\alpha, \xi)$ has logarithmic characteristics. To construct quadrature formulas we use the approach known in science. Smooth function $\sigma(\xi)$ we describe with linear, piecewise continuous functions:

$\sigma(\xi)=\sum_{n} \sigma_{n} S_{h}\left(\xi-\alpha_{n}\right)$

where $S_{h}(\alpha)=\left\{\begin{array}{rl}1-\frac{|\alpha|}{h}, & |\alpha| \leq h, \\ 0, & |\alpha|>h ;\end{array} \sigma_{n}=\sigma\left(\alpha_{n}\right) ; \alpha_{1}, \alpha_{2}, \ldots, \alpha_{N}\right.$ nodal points covering the region $[a, b]$; moreover $\alpha_{j+1}=\alpha_{j}+h$ $a$ - partitioning step. Substituting (9) into the integral:

$I(\alpha)=\int_{a}^{b} \sigma(\xi) J(\alpha, \xi) d \xi$

we obtain quadrature formulas:

$I\left(\alpha_{v}\right)=\sum_{n=0}^{N} A_{v, n} \sigma_{n}, v=\overline{0, N}$

where:

$A_{v, n}=\int_{-h}^{h} S_{h}(t) J\left(\alpha_{v}, \alpha_{n}+t\right) d t$

Based on formulas (11) and (12) the system of equations and inequalities (8) can be written as:

$$
\left\{\begin{array}{l}
\sum_{n=0}^{N+2} A_{v, n} \sigma_{n}=F_{v}, \sigma_{v}<0, \\
\sum_{n=0}^{N+2} A_{v, n} \sigma_{n} \leq F_{v}, \sigma_{v}=0, \\
\sum_{n=0}^{N} \lambda_{n}^{1} \sigma_{n}=P_{x}, \sum_{n=0}^{N} \lambda_{n}^{2} \sigma_{n}=P_{y}, v=\overline{0, N} ;
\end{array}\right.
$$

where: $A_{V, N+1}=f_{1}\left(\alpha_{V}\right), A_{V, N+2}=-f_{3}\left(\alpha_{V}\right), F_{V}=-\tilde{f}\left(\alpha_{V}\right)$, $\lambda_{n}^{1}=\int_{-h}^{h} S_{h}(t)\left(\tilde{R}_{y}\left(\alpha_{n}+t\right) \cos \left(\alpha_{n}+t\right)-\rho \tilde{R}_{x}\left(\alpha_{n}+\right.\right.$ $\left.t) \sin \left(\alpha_{n}+t\right)\right) d t, \quad \lambda_{n}^{2}=\int_{-h}^{h} S_{h}(t)\left(\tilde{R}_{x}\left(\alpha_{n}+t\right) \sin \left(\alpha_{n}+\right.\right.$ $\left.t)-\rho \tilde{R}_{y}\left(\alpha_{n}+t\right) \cos \left(\alpha_{n}+t\right)\right) d t$.

It marked $\sigma_{N+1}=D_{x}, \sigma_{N+2}=\theta$.

Then the system (13) is reduced to quadratic programming problem (Maksimovic et al., 2012), namely the problem of minimizing the magnitude $\mathrm{Y}=\sum_{v=0}^{N+2}\left(\sum_{n=0}^{N+2}\left(A_{v, n} \sigma_{n}-F_{v}\right) \sigma_{v}\right)$ under conditions:

$$
\left\{\begin{array}{l}
\sum_{n=0}^{N+2} A_{v, n} \sigma_{n} \leq F_{v}, \text { de } v=\overline{0, N}, \\
\sum_{n=0}^{N} \lambda_{n}^{1} \sigma_{n}=P_{x}, \sum_{n=0}^{N} \lambda_{n}^{2} \sigma_{n}=P_{y}, \\
\sigma_{n} \leq 0, \text { де } n=\overline{0, N} ;
\end{array}\right.
$$

where it is assumed that $A_{N+1, n}=A_{N+2, n} \equiv 0, n=\overline{0, N+2}$. This problem is solved numerically using methods known in the studies.

Contact area using the above algorithm can be found directly. Solving the problem (13) and finding the appropriate distribution of contact stresses $\sigma_{n}$, we obtain that the points where $\sigma_{n}<0$ determine the contact area, and have the points where $\sigma_{n}=0$ determine the areas that are free from stress.

At sufficiently high accuracy of the proposed algorithm indicate solutions of problems given in Maksimovic et al. (2012). In particular, for a circular punch from the above given examples, it follows that with the choice of a sufficiently great number of nodal points can be obtained solution with pre-specified accuracy.

\section{CONTACT STRAINS UNDER THE STAMP, THE SHAPE OF WHICH MATCHES THE SHAPE OF THE HOLE}

Let first we have the case of isotropy, for which executed basic equations for generalized plane stress state and the equation of the hole is given by ratio (5). Then in parametric form, it will look like:

$x=\tilde{r}_{x}(1-\delta \cos (k \alpha)) \cos \alpha, \quad y=\tilde{r}_{y}(1-\delta \cos (k \alpha)) \sin \alpha$, $\alpha \in[-\pi, \pi)$.

For simplicity, we assume that $E_{1}=E_{2}=E=1.25$; $G=0.5 ; \quad v_{12}=v_{21}=v=0.25 ; \quad \rho=0 ; \quad Q_{y}=-\frac{P_{y}}{2 G R}=0$ ( $R=\frac{R_{x}+R_{y}}{2}$ ) and $\delta=0$ (i.e., the surface of the hole is not wavy).

The results of calculations of contact stresses with $\chi=2.2$; $N=601$ i $D=\left[-100^{\circ}, 100^{\circ}\right]$ are shown in Fig. 2 (as in work Maksimovic et al. (2012)). For different directions of the main force vector are obtained different distributions of contact stresses. For curves (1-4) force acts in the direction of major axis, and for curves (1'-4') - in minor axis direction. Here curves (1 and $\left.1^{1}\right)$ correspond to the principal vector of force $Q_{x}^{1}=-\frac{P_{x}^{1}}{2 G R}=$ 0.005 , curves (2 and 2') $-Q_{x}^{2}=0.01$, curves (3 and $\left.3^{\prime}\right)$ $Q_{x}^{3}=0.015$, curves (4 and 4') $-Q_{x}^{4}=0.02$.

As seen from this figure, in the case of force acts in the direction of major axis maximum stress is reached in the center of the contact area. When the action efforts act in the minor axis direction (Fig. 2b) maximum in the distribution of contact stresses is achieved at the points $\alpha=\mp 55^{\circ}$, while it at the appropriate value of the force is more than 2 times smaller than in Fig. 2a. In addition, in two cases there is almost a linear relationship between the maximum values of the stresses and the main force vector. Contact area in all the cases coincides and is $\approx\left[-84^{\circ}, 84^{\circ}\right]$. 

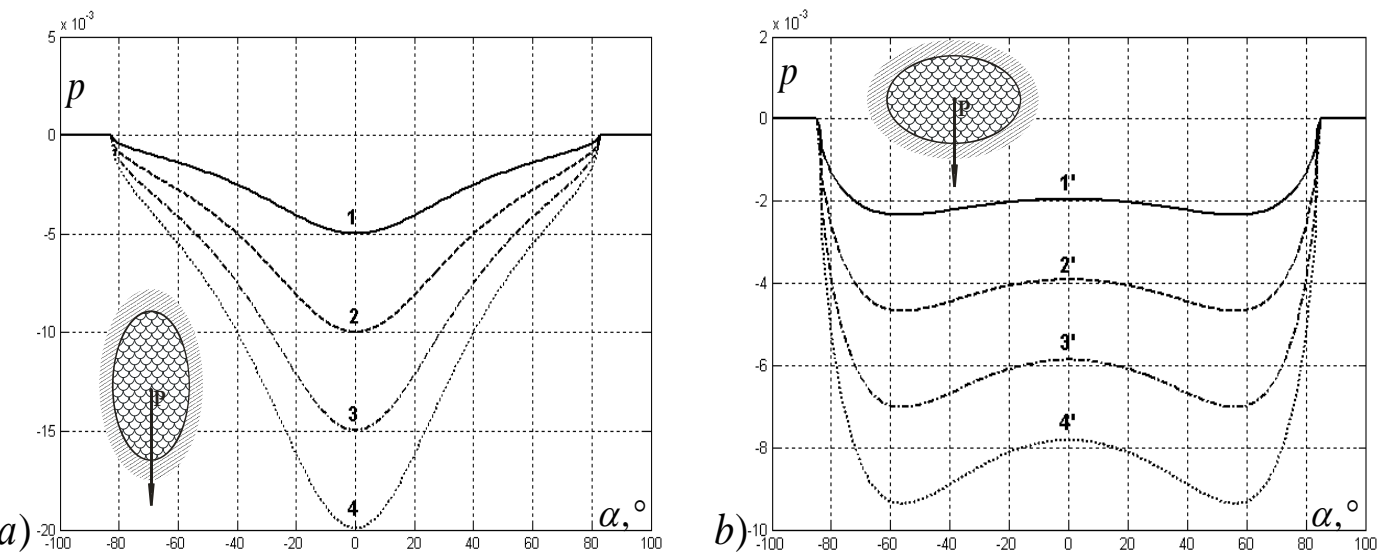

Fig. 2. Contact stresses under stamp of elliptical form: a) force acts in the direction of major axis; b) force acts in the direction of minor axis

Further, we assume that there is a orthotropy for which basic equations for generalized plane stress condition are performed. Unlike previous abstract example, consider specific application cases of orthotropic materials. For example, first we have aluminum borosilicate fiberglass (E-glass), which is characteristic for isotropic properties in the plane, which is perpendicular to the direction of the fibers. For this material we have:

$E_{x}=6.25 \mathrm{GPa} ; E_{y}=21.2 \mathrm{GPa}$,

$G_{x y}=9 \mathrm{GPa}, v_{x y}=0.251$

From the characteristics (14) we pass to dimensionless characteristics:
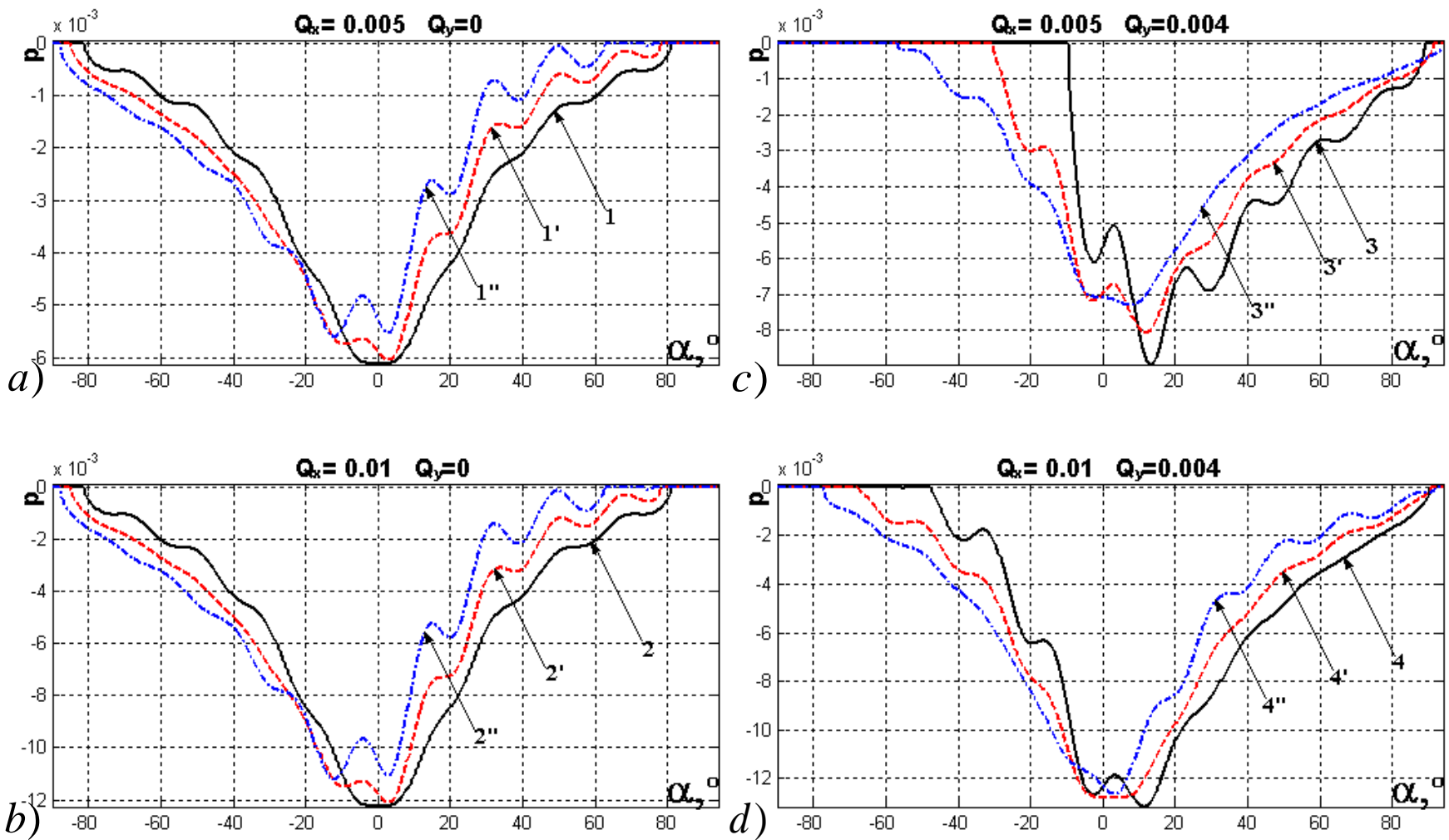

Fig. 3. Contact stresses under the stamp, which shape is similar to the elliptical on the surface of a similar hole in the orthotropic plate (E-glass), when bigger force acts in the direction of major axis

$$
\begin{aligned}
& E_{1}=\frac{E_{x}}{2 G_{x y}}=3 \frac{17}{36}, E_{2}=1 \frac{8}{45}, G=0.5, v_{12}=0.251, \\
& v_{21}=0.251 \cdot \frac{E_{2}}{E_{1}}=0.251 \cdot \frac{212}{625}=0.0851392 .
\end{aligned}
$$

The results of calculations of contact stresses, if $\delta=0.0004$ $k=20$ (there is waviness of surfaces of the hole and stamp), $N=461, D=\left[-90^{\circ}, 94^{\circ}\right]$, at different values of the coefficient of friction $\rho$ and the main vectors of forces $Q_{x}$ та $Q_{y}$ are shown in Fig. 3 and 4. Curves (1-4) in both figures correspond to the coefficient of friction $\rho=0$, curves $\left(1^{\prime}-4^{\prime}\right)-\rho=0.2$, and curves $(1 "-4 ")-\rho=0.4$. 

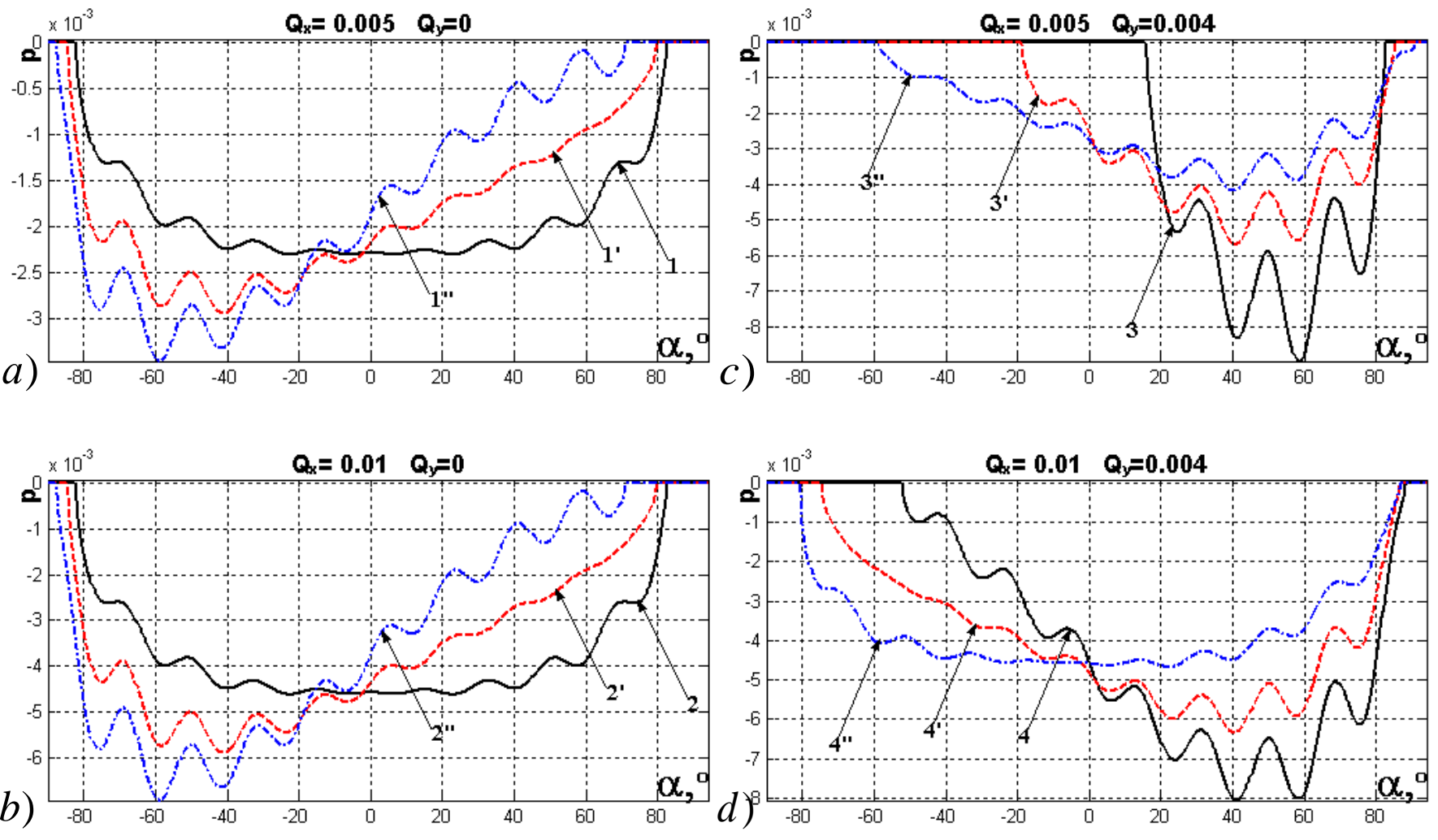

Fig. 4. Contact stresses under the stamp, which shape is similar to the elliptical on the surface of a similar hole in the orthotropic plate (E-glass), when bigger force acts in the direction of minor axis

As shown in Fig. 3 and 4 increase of the coefficient of friction leads to increase of the asymmetry in the negative (clockwise) direction, and an increase relatively to the main vector of force $Q_{y}$, however, increases the asymmetry in the positive direction. Let investigate dependence of the characteristics of elasticity and relative distribution of contact stresses. To do this, choose the case where $Q_{x}=0.01, Q_{y}=0.004 \mathrm{i} \rho=0.2$.
The results of calculations of contact stresses with $G=0.5$ are shown in Fig. 5 and 6 for orthotropic materials such as E-glass (properties (14')) - curve (1), fiber reinforced with boron $\left(E_{1}=\right.$ $12 \frac{7}{17}, E_{2}=1 \frac{41}{170}, v_{12}=0.35, v_{21}=0.035-$ curve (2), fiberreinforced with graphite $\left(E_{1}=18.625, E_{2}=0.75, v_{12}=0.31\right.$, $\left.v_{21}=\frac{93}{7450}\right)$ - curve (3).

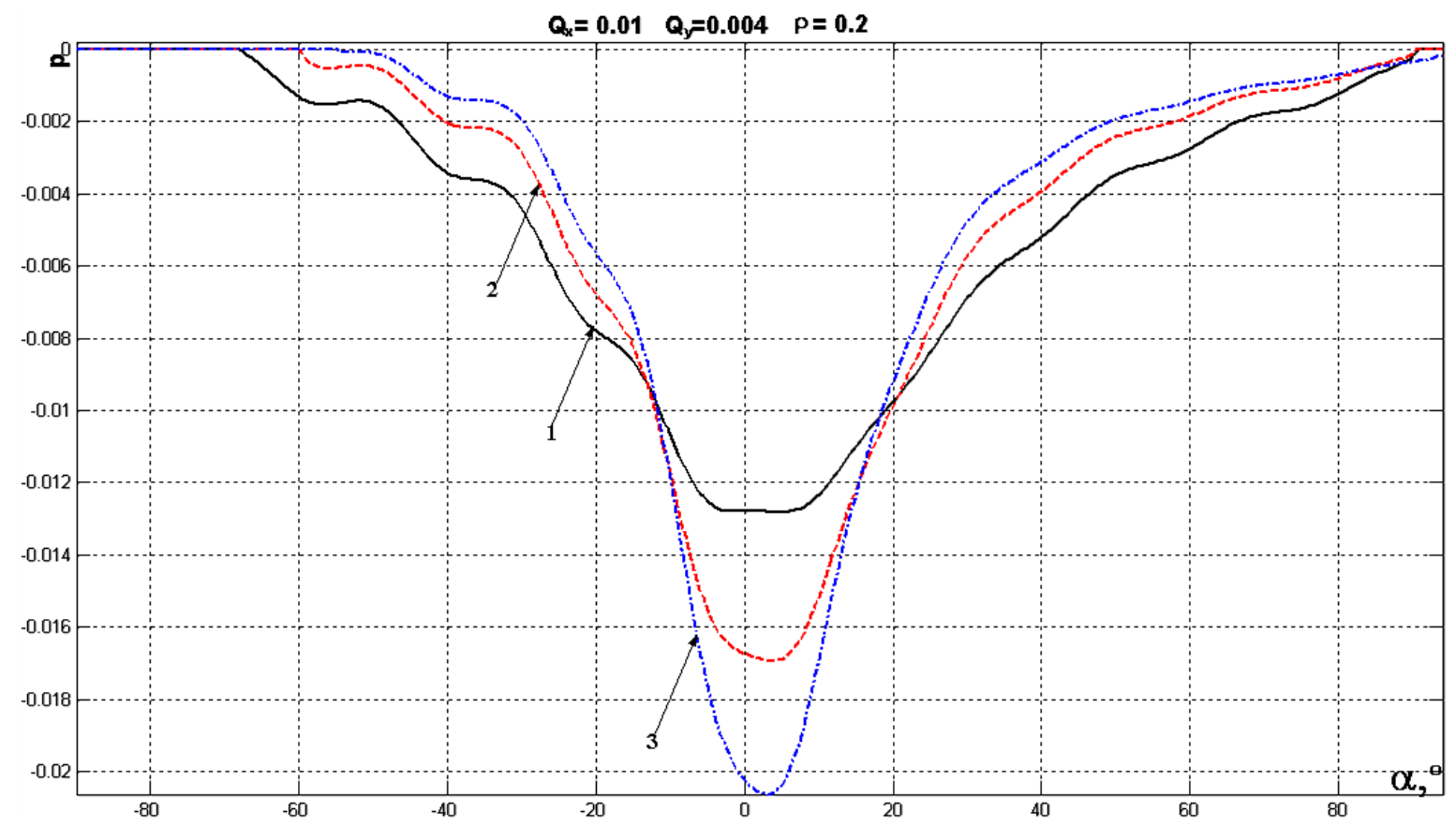

Fig. 5. Contact stresses under the stamp, which shape is similar to the elliptical, on the surface of a similar hole in different orthotropic plates when a larger force acts in the direction of major axis 


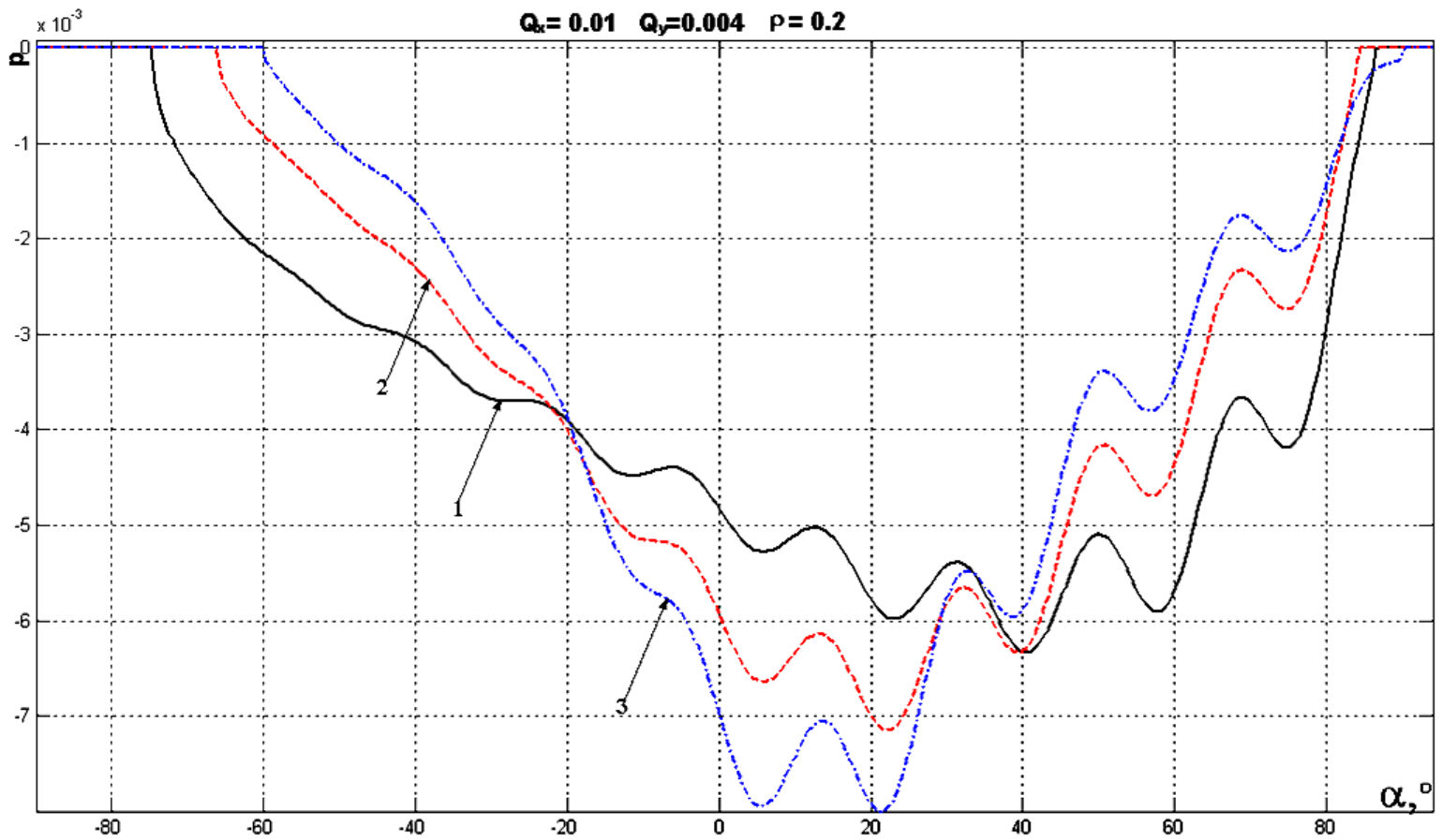

Fig. 6. Contact stresses under the stamp, which shape is similar to the elliptical, on the surface of a similar hole in different orthotropic plates when a larger force acts in the direction of minor axis

As shown in Fig. 5 and 6 in the distribution of contact stresses relative size of the contact area depends on the Poisson ratio (the smaller $v_{12}$ is the greater the contact area) which indicates the physical meaning of the Poisson ratio. While it is clear that the contact area and maximum stress significantly influence the modulus of elasticity. For a more overall understanding of the influence it'd be better to consider more examples and without waviness. In this study, we aimed to describe the versatility of the developed numerical and analytical methods for which is of no value bulkiness of recording of the resulting integral equation and the complexity of analytically solving of the problem $(7,4)$.

\section{CONCLUSIONS}

Numerical and analytical methods for calculating the relative distributions of contact stresses on the surfaces of complex shape holes in infinite plates (Maksimovic et al., 2012), which is based on the methods of boundary integral equations, mechanical quadratures and quadratic programming, which is developed for the case of orthotropy are worked out. Integral equations were constructed similarly as in Bozhydarnik et al. (2007); Kombel (2003); Panasyuk and Tepliy (1975), Savin (1968), Syackyj and Shynkarchuk (2010, 2011); Syackyj and Trokhymchuk (2009, $2010 \mathrm{a}, \mathrm{b})$. The very same methods are tested on simpler problems (Maksimovic et al., 2012), for which the analytical solutions are known in the science.

\section{REFERENCES}

1. Abd-El-Naby S.F.M., Hollaway L., Gunn M. (1993), The tangential stresses in an infinite orthotropic composite plate with a loaded hole Original Research Article, Composites Engineering, Vol. 3, Issue 1, 19-30.

2. Bozhydarnik V.M., Kotsyuba A.Yu., Maksimovic O.V. (2007), Contact interaction of arbitrary shape stamp and plate with elliptical hole, Bulletin of Dnipropetrovsk University Series: Mechanics, Edition 11, Vol. 2, No 2/2, 29-34, (in Ukrainian).

3. Kombel S.M. (2003), Landing of hard drive into elliptical hole of infinite orthotropic plate, Engineering, № 8, 29-34, (in Ukrainian).

4. Maksimovic V.M., Kotsyuba A.Yu., Lavrenchuk S.V. (2012), Plane contact problems of elasticity theory for bodies of complex shape, LNTU, Lutsk, (in Ukrainian).

5. Panasyuk V.V., Tepliy M.Y. (1975), Some contact problems of the theory of elasticity, Naukova Dumka, Kyiv, (in Ukrainian).

6. Savin G.H. (1968), The stress distribution around the holes, Naukova Dumka, Kyiv, (in Russian).

7. Syackyj A.O, Shynkarchuk N.V. (2010), Mixed contact problem for orthotropic plate with elliptical hole and rigid disk, Herald of Ternopil National Technical University, Vol. 15, No 4, 7-13, (in Ukrainian).

8. Syackyj A.O, Shynkarchuk N.V. (2011), Mixed contact problem for isotropic plate with curvilinear hole and rigid disk, Herald of Ternopil National Technical University, Vol. 16, No 1, 16-21, (in Ukrainian).

9. Syackyj A.O, Trokhymchuk O.Ya. (2009), Pressure rigid stamp with corner points on a partially strengthened contour elliptic hole in infinite orthotropic plate, Herald of Ternopil State Technical University, Vol. 14, No 3, 12-18, (in Ukrainian).

10. Syackyj A.O, Trokhymchuk O.Ya. (2010a), Mixed contact problem for a plate with a curvilinear hole and the system of stamps with corner points, Herald of National Technical University of Ukraine "Kyiv Polytechnic Institute". Series of "Mechanical Engineering", No 58, 36-41, (in Ukrainian).

11. Syackyj A.O, Trokhymchuk O.Ya. (2010b), Pressure system of two stamps with corner points on a partially strengthened contour elliptic hole orthotropic plate, Herald of Ternopil State Technical University, Vol. 15, No 1, 14-20, (in Ukrainian). 